\title{
Simulacro Made in China: Relatos e Origem da Imagem em Transformação
}

\author{
Simulacra Made in China: Reports and Origin of the Image \\ in Transformation
}

\section{RESUMO}

Ideias, linguagem e suas formas de expressão representam culturas pela configuração da narrativa do homem em suas conquistas. $\mathrm{O}$ conhecimento orienta a capacidade técnica e prática para as relações sociais, todavia, para domínio de todas as faculdades do conhecimento que tem sentido do Ocidente ao Oriente. Sobretudo, considerando que importantes descobertas da história da civilização também surgiram do Oriente. Nas artes e na literatura, a China contribuiu de forma muito significativa não só para o Ocidente, mas, também, para o patrimônio artístico da humanidade, principalmente durante o século $\mathrm{XX}$, quando os ideais político-sociais eram representados pela arte moderna, encontrando referências estéticas e formais no Oriente. Por exemplo, o significado da obra de Brecht, diante de divisões político-ideológicas no século passado, no que concerne à arte e suas técnicas para representação. Porém, não como instrumento ideológico, mas pela sua estética, que, por diversos motivos e, também, único e universal, o fascínio do homem pelo novo. E, o mesmo fascínio para transformá-lo, parte dele ser ou ter, enquanto que, por meio de sua arte, o representa. Enfim, realizações aproximam culturas ocidentais e orientais, ao mesmo tempo em que as distanciam ao se revelarem, uma na outra, características próprias para discerni-las.

Palavras-chave: Técnica. Arte. Cultura.

\section{ABSTRACT}

Ideas, language and their forms of expressions represent cultures by the configuration of man's narrative and his achievements. Knowledge guides the technical capacity and practice for the social relations; however, to the domain of all the knowledge faculties that makes sense from the Occident to the Orient. Especially, considering that important History discoveries of the civilization also emerged from the Orient. China contributed in a very significant way to the arts and literature for the artistic humanity heritage. 
Mainly, during the 2oth century, when the social-politics ideals were represented by the modern art finding formal and aesthetics references in the Orient. For instance, the Brecht's work meaning, in front of the politic-ideological divisions on the last century, in what concerns to the art and its representation techniques. However, not as an ideological instrument, but for the aesthetic dimension, which is, for several reasons and universal, the fascination of new on the man. The same fascination to transform him, part of him to be or to have while represents him by means of his art. Finally, realizations approximate occidental and oriental cultures at the same time that distance them showing one on the other their own characteristics to discern them selves.

Keywords: Technique. Art. Culture.

\section{INTRODUÇÃO}

Em nossos dias, tanto o Ocidente como o Oriente se apresentam frente à inovação tecnológica ou à difusão de ideias de forma muito semelhante. Porém, se o Oriente é contemplado pelo Ocidente e vice-versa, algumas diferenças são percebidas como resultado dessa observação. Evidentemente, cada qual constataria suas particularidades em suas características únicas diante de uma distinção. E cada vez mais, percebe-se a aproximação das culturas, o que não significa a unificação. Deste modo, intensificam-se as diferenças, justamente pela diversidade cultural. A importância da presença da China nos dias atuais deve-se não só à dinâmica de uma economia de mercado, à convergência tecnológica e digital facilitando a comunicação e o acesso a esta cultura, mas, sobretudo, como um fenômeno quase recíproco; não totalmente, devido ainda ao controle que o governo chinês exerce sobre a imprensa, censurando o ideal de liberdade de expressão, mesmo com constantes manifestações e protestos contra esse controle in patria e nas redes sociais. Porém, ainda novas relações comerciais e formas diferentes de relações de produção distinguem as condições do indivíduo em uma sociedade globalizada. Seja o lucro como objetivo ou a luta pela dignidade humana, o que se discute internacionalmente sobre a humanidade é a erradicação da pobreza, o comércio justo e o desenvolvimento sustentável. Metas são estabelecidas para realizações. A criatividade e a inovação são primordiais para atenderem aos ideais que, por fim, seriam concretizados e configurados em novas imagens que continuariam em transformação. Em síntese, mantendo absoluta a arte, pela contemplação, constatação, discordância ou compreensão pelo Oriente e Ocidente, ou melhor, de um pelo outro. Em vista disso, do Ocidente para o Oriente, diretamente a China, lembremos que o acesso dos ocidentais a esse país foi, por muito tempo, dificultado pela distância geográfica, além de o país ser marcado por períodos de grandes diferenças em relação ao mundo ocidental, pela indústria e sua cultura que se mantiveram fechadas para o resto do mundo e autofocadas [15]. Hoje, com os museus, a internet e os meios de comunicação, conhecemos melhor a cultura chinesa e seu universo artístico. Assim representada no Ocidente, é, em seus aspectos culturais, importante na influência da imagem e principal elemento de sustentação da produção em massa e da globalização. Porém, no século XVIII, surge no Ocidente, com o conceito de Estética, a expressão Estética chinesa, com referência aos produtos que chegavam do país em questão. Os chineses, percebendo que 
seus produtos - principalmente em artes decorativas, pelas características diferentes e como objetos exóticos - eram de grande interesse para o mercado europeu, fortaleceram a respectiva imagem que o próprio Ocidente idealizou. Com essa imagem ainda mais forte, eles mantinham suas vendas, exportando, pois a Europa esperava que eles correspondessem a esta imagem.

\section{DISCUSSÃO}

A estética e a criatividade do Ocidente encontraram formas de diferenciar a realização pela novidade que encontravam na cultura chinesa. A arte chinesa tem suas formas próprias tanto na arquitetura, na pintura, na música como no teatro. Mas também no sentido próprio do termo, a Estética chinesa é a reflexão dos chineses sobre as artes e sua Filosofia da Arte e do Belo [18], também como disciplina na tradição chinesa. No vocabulário de Estética, encontramos registros da Introdução à pintura de paisagens, de Tsong Ping, do século V d.C. - sem nos aprofundarmos na vasta história da China, mas apenas com o propósito de indicar a importância das artes na formação da personalidade chinesa, da sua relação com o mundo, tanto na experiência da estrutura do universo como na influência mágica sobre o mundo. Sobretudo o valor da arte na relação com as estruturas sociais e uma concepção mais recente com princípios marxistas. Porém, a conjuntura do mundo vem tomando, nas últimas décadas, uma dimensão diferente. No século XIX a Europa já dominava alguns países asiáticos. Com a Revolução Industrial e o consequente desenvolvimento do capitalismo, os europeus estabeleceram uma hegemonia desses países sobre os demais, que ainda estavam em processo de desenvolvimento. O melhor exemplo certamente está nas relações comerciais e no controle econômico dos países europeus hegemônicos sobre os países ainda em desenvolvimento, que naquela época, era o caso da China.

Noções elementares de uma dinastia, que antecede em séculos a idade de Cristo, para o avanço tecnológico e desenvolvimento de uma economia que tomou, cada vez mais, uma dimensão de poder para o Ocidente. Um dragão, que, além de um ideal, tornou-se referência real aos olhos do mercado mundial. Um espetáculo que ultrapassa os objetivos mercantis e, "à sua imagem", configura o simulacro made in China.

A dimensão das artes chinesas como simulacro na tradição ocidental e imagem configurada tem, a partir das primeiras décadas do século passado, notoriedade pela dramaturgia de Bertolt Brecht, com a "técnica do distanciamento" * [18], mas esta sendo apenas original em integrar aquelas em suas obras. Por um lado, a imagem se configura, prevalecendo o momento (tempo) e a oportunidade (espaço) para caracterizar a realização e domínio público do que possa ser diferente e, talvez, inovador. A novidade como qualidade abre outra discussão, mas a ideia de inovação é sempre associada a de uma ação como inovação pelo que se propõe nesta reflexão $[19,20]$.

Por outro lado, constata-se que no passado, durante o período clássico, tanto na tragédia como na comédia, todas as técnicas que comportavam essas mesmas características, pela tradição, em relação às convenções, tinham um sentido de distanciamento; método do teatro épico em oposição ao teatro dramático, que envolvia o espectador na ilusão.
* A noção de distanciamento está relacionada com a dramaturgia de Brecht, mesmo que ela seja, atualmente, utilizada fora desse contexto. Não se trata apenas de uma alusão a um estilo de jogo ou de técnicas específicas como vemos frequentemente, mas de evocar toda a concepção do "teatro épico", ou seja, da submissão à necessidade de uma distância, do distanciamento ou ausência da ilusão em todos os níveis. Primeiramente, pela dramaturgia épica, não se objetiva a identificação do ator com o personagem. Em seguida, em oposição ao teatro dramático, a intenção é romper com qualquer ilusão, entre espetáculo e espectador ou qualquer identificação entre eles, por meio de técnicas, privando-os da continuidade das cenas sensacionalistas, permitindo dessa forma ao público a reflexão e uma consciência com objetivo político. 
Figura 1 - Detalhes do filme The great dictator, de Charles Chaplin (EUA, 1940). Fonte: Disponível em: $<$ http://djardine.blogspot. com/2010/07/great-dictatorusa-1940-charlie-chaplin. html>. Acesso em: 10 mar. 2012.

Duas teorias centrais na análise das imagens, ambas versam sobre a polêmica dicotomia ilusão e real, desde sempre. Porém, mesmo que nos reportemos às origens, ainda de forma generalizada a nos situarmos na busca do "verdadeiro" sentido, isto é, encontrar uma lógica para a configuração da imagem na contemporaneidade. Visto que, pelos estudos de Walter Benjamin (1892-1940) [8, 9, 10, 11, 12, 13] sobre a percepção, o papel da aura em relação à reprodutibilidade técnica e, também, pelas análises sociológicas de Jean Baudrillard [3, $4,5,6,7]$, notamos uma grande influência das obras de Bertolt Brecht [17] no pensamento desses teóricos. Mas, com grande importância, deve-se considerar que Brecht não era comunista. Mas, pelas influências das obras de Hegel e Marx em suas obras na década de 1930, ele tinha ideais que podiam ser entendidos como "marxistas" sem que tivesse alguma militância política no comunismo ou partidária. Porém, aqui, o importante é salientar a importância das obras de Hegel e de Marx na concepção de suas obras. Nessa época, Walter Benjamin frequentava os mesmos ambientes que Brecht, principalmente em Berlim e, como colegas, participavam das discussões sobre política, sociedade, desenvolvimento industrial, o papel da cultura e principalmente, a produção cultural: o teatro, a fotografia, o rádio, o cinema, a publicidade e os meios de comunicação. De outra forma, pela pesquisa e interesse sociocultural, o teórico francês Jean Baudrillard, também germanista, se interessa pela obra de Brecht e as traduz do alemão para o francês. A coincidência de muitas das abordagens sobre os aspectos relacionados à percepção do indivíduo, sociedade de consumo em face à semiologia e todos os aspectos sedutores da "cultura industrial", remete não só a um mesmo método de análise crítica semiológica como também permite, pela teoria, identificar, antes mesmo da "técnica do distanciamento" de Brecht, a importância que a obra $O$ capital de Marx, teve, ao constatarmos reflexões sobre a "mais-valia” e valores de uso e troca. Reflexões específicas, delimitando-se ao entendimento da distância entre a realidade sócio-histórica, por meio do distanciamento em relação à arbitrariedade do signo, como distância que deve existir entre o significado e significante. Como representação cênica, Étienne Souriau [18] apresenta um esclarecimento como modelo da "técnica do distanciamento", que é a transposição da história de Hitler e do Terceiro Reich para o universo do circo, procurando distância nas passagens das cenas com humor, principalmente, como fez Chaplin [2].
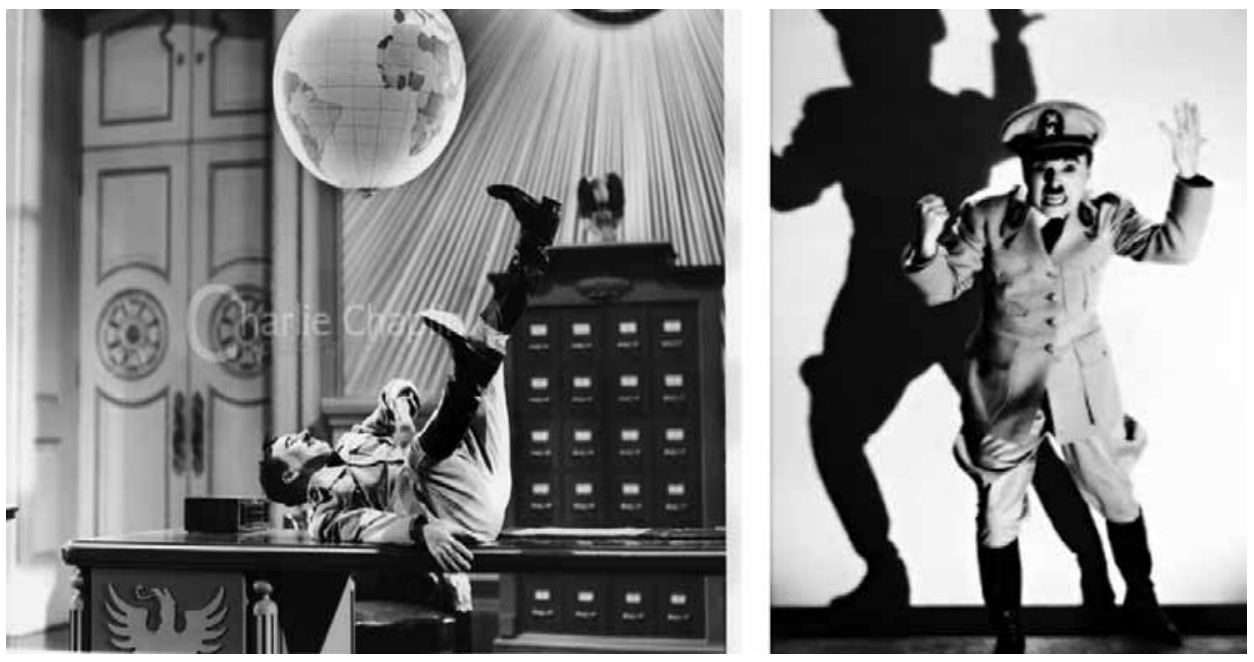
O distanciamento revela sempre uma crítica. Segue-se o modelo da dialética materialista. Os elementos se distinguem em uma relação de oposição, mas se apresentam juntos e significam a estrutura da representação, com formas de interrupção na continuidade, dando a impressão de montagens oferecendo uma distância entre as cenas. A obra tem um modo específico de identificar o real, eliminando a ilusão e o espectador precisa também decifrar e entender esse modo de leitura. Porém, em relação à ilusão como teoria, de forma sistemática e contrária à “teoria do distanciamento” ** [18], observa-se a ambiguidade da relação do espectador com o espetáculo, conforme enunciado por Étienne Souriau [18], ao mostrar que o espectador pode acreditar e não acreditar no que está vendo, "l'illusion est également une convention, une règle de jeu que le spectateur veut bien accepter"***.

No ensaio de Jean Baudrillard, intitulado L'illusion cinématographique perdue, ele questiona a possibilidade da ausência de ilusão pela linguagem cinematográfica em sua evolução e progresso técnico. Na proporção de um avanço tecnológico, passando do cinema mudo ao falado, do preto e branco e em cores até a alta tecnologia. Para esse pensador, é nessa alta definição cinematográfica que a ilusão se torna ausente. Afirma ele que o cinema atual não conhece mais nem a alusão nem a ilusão. O espectador se encontra em um estado hipertécnico, hipereficaz, hipervisível sem deixar espaço, silêncio, perdendo a especificidade das imagens em direção a uma tecnologia de alta definição no sentido de uma perfeição inútil da imagem. Esclarecendo melhor, o autor diz [7]: "Plus on approche de la définition absolue de la perfection realiste de l'image, plus se perd sa puissance d'illusion" ****.

Quando Baudrillard, na conclusão de seu ensaio, afirma que nós esquecemos o que a modernidade nos ensinou, a probabilidade de que ele faz alusão ao distanciamento é grande, pelos motivos anteriormente citados, sobre seu interesse pela obra de Brecht e naturalmente, pela sua própria obra, indicando que é a subtração que oferece a força e que da ausência nasce a energia. Do ponto de vista dos valores da modernidade, podemos perceber na obra de Brecht, "depois de conhecer a atuação do artista chinês Mei Lan Fang e quando trabalha o efeito de 'estranhamento' da arte chinesa, que consistia em criar um deslumbramento" [14].

\section{CONCLUSÃO}

A arte tem seus valores em si mesma, que são estéticos (aisthésis) ou técnicos, e avalia outros valores apenas em função de si própria. A desproporção entre os valores estéticos e os não estéticos (anaisthésis) está frequentemente associada às disparidades sociais. Em todas as épocas, os artistas ou amadores formam classes distintas na sociedade, levando a uma diferença de valores estéticos ou não estéticos e a uma visão hostil pela realização de algumas obras, independentemente do grau de civilidade e de culturas [16]. Vêem-se, por exemplo, realizações com propósitos nacionalistas. Contudo, sempre a mesma obra é julgada de diferentes formas por diferentes indivíduos e, sobretudo, em diferentes épocas. Como identificar evolução da arte diante desse fato? Diferente de outras grandes evoluções humanas, em estética não existe evolução, e por meio de seu objeto que é a arte, contatamos.

Simulacro Made in China: Relatos e Origem da Imagem em Transformação
** As teorias de distanciamento concernem ao que se estuda sobre a técnica do distanciamento na dramaturgia. Pelos estudos de Étienne Souriau, constatamos que a técnica do distanciamento existe há muito tempo. Brecht as conheceu no teatro chinês e pelos gêneros de espetáculos não lineares como o circo e o music-hall. Mas podemos entender que Brecht teve sua originalidade ao integrar em sua dramaturgia a sua "teoria do distanciamento", incorporando-a ao seu sentido amplo. Entretanto, podemos verificar que os espetáculos do período do classicismo e de tradição apresentaram técnicas desse gênero. $E$, tudo o que se reporta às "convenções" tem um sentido de distanciamento. E mesmo com o naturalismo, uma reação se formou e, procurou-se remontar os grandes períodos da arte dramática. Lembremonos o uso de versos na tragédia e na comédia, o "distanciamento" do herói trágico (particularmente, nas peças de Racine), e tudo que resulta do monólogo ou da aptidão cênica com relação ao público. Recordemos ainda, o papel do coro na tragédia da Antiguidade, que, segundo Schiller e Nietzsche, separa a reflexão da ação, rompendo com a ilusão, o que nos mostra uma aproximação com a técnica de Brecht e sua "teoria do distanciamento". Pois, com o coro interrompendo a ação, o sentido é o de comentar e oferecer uma reflexão. Entre outras formas de teatro, como o de estrada ou a commedia dell'arte, é que, também, encontramos técnicas desse gênero; porém, em suas formas mais puras. 
*** "A ilusão é igualmente uma convenção, uma regra de jogo que o espectador bem quer aceitar." (Tradução nossa).

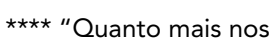
aproximamos da definição absoluta, da perfeição realista da imagem, mais se perde o poder de ilusão." (Tradução nossa).

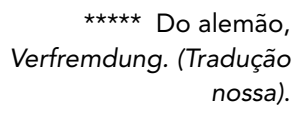

Figura 2 - Detalhes da atuação de Mei Lan Fang no teatro chinês. Fonte: Brecht: Die Kunst zu leben. Produção de Joachim Lang, 2006.

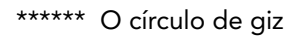
caucasiano. Existe tradução para a língua portuguesa.

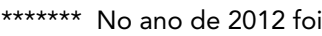
realizado o evento Ano da Cultura Chinesa na Alemanha.
Porém, retornando às formas de representação, especialmente considerando a catharsis [1], a partir da modernidade, devemos observar que as "técnicas do distanciamento" empregadas por Bertolt Brecht sempre existiram. Justamente, pelo teatro chinês e os gêneros de espetáculos não lineares, como o circo, é que eram empregadas as "técnicas do distanciamento". Assim, Brecht, em 1941, quando de sua viagem por Moscou, se impressionou com o desfile de $1^{\circ}$ de Maio e comemorou a inauguração do metrô com um poema, como também contemplou com grande interesse uma apresentação do ator de teatro chinês Mei Lan Fang, que atuava também como mulher. Nesse momento, podemos entender quando Brecht teve uma experiência estética e na qual, possivelmente, por meio de "alusão e ilusão” - segundo as observações de Baudrillard em relação ao teatro chinês, a Ópera de Pequim -, ele encontra outras formas criativas para o seu teatro. Brecht escreveu que, ao assistir a Mei Lan Fang, percebeu o efeito de "estranhamento"***** na arte chinesa, declarando que esse estranhamento consistia em criar um deslumbramento. Percebe-se com esse relato a sua experiência estética frente àquela obra.
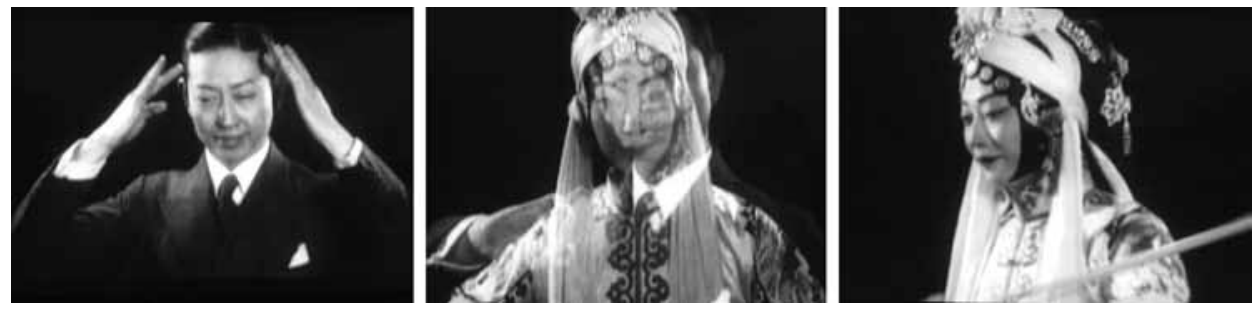

Experimentou um momento em que o conhecimento ocidental se encontrava com o oriental por meio das técnicas chinesas de espetáculos, configurando a imagem. Brecht

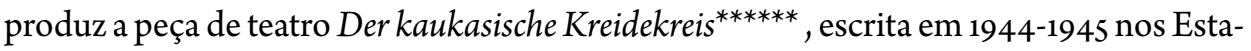
dos Unidos e, em 1954, apresentada em Berlim. Nos dias de hoje, podemos experimentar relações culturais com uma narrativa visual, por exemplo, em Wiesbaden quando, nos dias 22 e 23 de maio de $2012^{* * * * * * *}$ foi reapresentada a referida peça no Hessisches Staatstheater, em cooperação com Chongqing Sichuan Opera Theatre.

Figura 3 - Detalhe da peça de Bertolt Brecht, Der kaukasische Kreidekreis, encenada pela Chongqing Sichuan Opera Theatre. Fonte: Disponível em: < http://www. cn2012de.com/deactivityall. aspx?type $=$ add \&tid $=12>$. Acesso em: 24 mai. 2012.

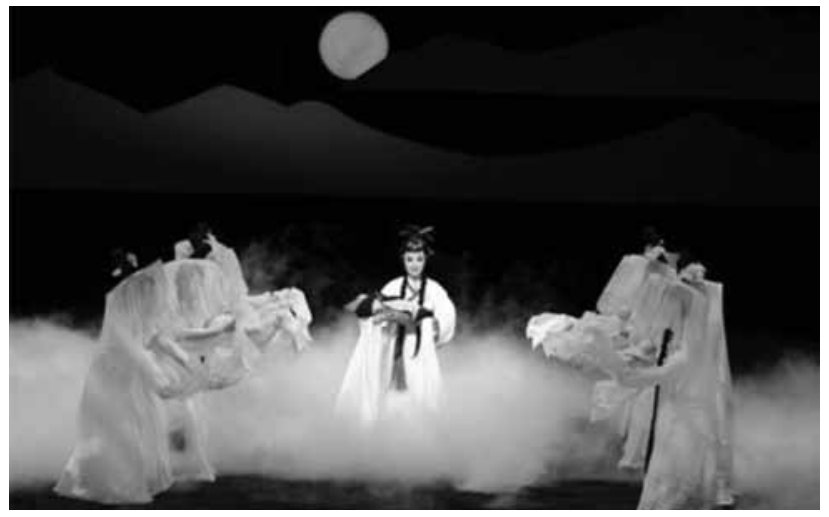




\section{REFERÊNCIAS BIBLIOGRÁFICAS}

[1] ARISTOTE. Poétique. Paris: Gallimard, 1996.

[2] ALBERSMEIER, F.-J. Texte zur Theorie des Films. Stuttgart: Reclam Verlag, 2003.

[3] BAUDRILLARD, J. Le système des objets. France: Gallimard, 1968.

[4] BAUDRILLARD, J. La société de consommation: ses mythes ses structures. Paris: Denoël, 1970.

[5] BAUDRILLARD, J. Simulacres et simulation. Paris: Galilée, 1981.

[6] BAUDRILLARD, J. Pour une critique de l'economie politique du signe. Paris: Gallimard, 1972.

[7] BAUDRILLARD, J. Illusion, désillusion esthétiques. Paris: Sens \& Tonka, 1997. p. 11.

[8] BENJAMIN, W. L’ouvre d'art à l'époque de sa reproductibilité technique. Paris: Allia, 2009.

[9] BENJAMIN, W. Paris, capitale du XIXe siècle: le livre des passages. Paris: CERF, 2009.

[10] BENJAMIN, W. OEuvres. Paris: Gallimard, 2000. 3v.

[11] BENJAMIN, W. Sprache und Geschichte. Stuttgart: Reclam Verlag, 2005.

[12] BENJAMIN, W. Medienästhetische Schriften. Frankfurt: Suhrkamp, 2002.

[13] BENJAMIN, W. Essais sur Brecht. Paris: La fabrique, 2003.

[14] BRECHT: Die Kunst zu leben. Direção: Joachim Lang. Produção: Andreas Bareiß, Gloria Burkert, Vera Meyer-Matheis, Jo N. Schäfer e Martina Zöllner. Alemanha : TV 6o Filmproduktion GmbH, 2006. Suporte desconhecido. Originalmente concebido para a televisão.

[15] GOMBRICH, E. H. Histoire de l'art. London : Phaidon, 2006.

[16] JIMENEZ, M. L'esthétique contemporaine. Paris: Klincksieck, 2004.

[17] KNOPF, J. Beltolt Brecht. Stuttgart: Reclam Verlag, 2000.

[18] SOURIAU, É. Vocabulaire d'esthétique. Paris: PUF, 1990. p. 626-627.

[19] WAGNER, C. Estética: imagem contemporânea: análise do conceito de inovação. 2013. 300 f. Tese (Doutorado)-Faculdade de Arquitetura e Urbanismo, Universidade de São Paulo, São Paulo, 2013.

[20] WAGNER, C. Esthétique: l'image contemporaine: l'analyse du concept de l'innovation. 2013. 300 p. Thèse (Doctorat en Arts Plastiques, Esthétique et Sciences de l’Art)-Université Paris 1 Panthéon-Sorbonne, Paris, 2013.

\section{AGRADECIMENTO}

Ao Nikolas, meu filho, tradutor do Resumo para o inglês.

CHRISTIANE WAGNER, doutora pela Faculdade de Arquitetura e Urbanismo da Universidade de São Paulo (FAU-USP) e pela Université Paris 1 Panthéon-Sorbonne e pesquisadora da Université Paris 1 Panthéon-Sorbonne - email: christiane.wagner@malix.univ-paris1.fr. 